\title{
WiMAX Throughput Evaluation of Conventional Relaying
}

\author{
Pavel Mach'1, Robert Bestak ${ }^{1}$ \\ ${ }^{1}$ Department of Telecommunications Engineering, Faculty of Electrical Engineering, Czech \\ Technical University, Technicka 2, \\ 16627 Prague 6, Czech Republic \\ \{machp2, bestar1\}@fel.cvut.cz
}

\begin{abstract}
The paper analyses performance of IEEE 802.16 standard, also known as WiMAX, when relay stations are introduced. Thanks to this feature, coverage area and throughput of the system may be significantly enhanced. The article defines the increase of system capacity of the relay based cell deployment compared to a conventional single hop deployment of the same area size. Further, the parameters that have main impact on the system with relays and its capacity are determined.
\end{abstract}

Keywords: WiMAX, performance, throughput, Relay Station.

\section{Introduction}

In recent years, broadband wireless systems established themselves as one of the fastest growing and developing area in the field of telecommunications. The current trends and demands are to deliver multimedia services such as voice, video, high definition TV (HDTV) or interactive games with guaranteed Quality of Service (QoS). To support high quality multimedia services, a high data transmission are necessary. Since the most of the wireless systems operate in high frequency bands above $2 \mathrm{GHz}$ [1], [2], the transmitted signal is highly attenuated in comparison with low bands. As a consequence, the cell size is smaller and more Base Stations (BSs) is needed. Another drawback in using of high frequencies is big power transmission requirement. An efficient way how to reduce power consumption and extend the range offers Mesh and Relay networks that are distinguished by multihop communication between individual nodes. Furthermore, the capacity of systems using Mesh and Relay topology can be enhanced when compared to conventional single hop deployment.

According to [3], three types of Relay Stations (RSs) are defined; fixed, nomadic and mobile. The relays are in most cases build in, owned and controlled by service provider. An RS is not directly connected to wire infrastructure and has the minimum functionality to support multihop communication. Multihop based network may also improve system performance when cooperation relay technique is put to use. This is accomplished by sending information simultaneously via multiple different paths and combining the received information at the side of the receiver [4]. 
Two concepts how to integrate multihop communication into IEEE 802.16 standard are presented in [5]. The first concept follows a centralized approach, where the BS has full control over the relay-enhanced cell and RS may be very simply. The second concept follows a semi-distributed approach, where RS coordinates the associated Subscriber Stations (SSs) itself. In the second case, the MAC protocol complexity of RS is comparable to BS.

The rest of the paper is organized as follows. Section 2 defines the simulation model, its parameters and basic assumption. Moreover, the scenarios that are taken into consideration are depicted. In section 3 , the very process of simulation is described by path loss model and by method of throughput calculation. The following section provides simulation results in comparison of single and multihop deployment by means of system throughput. The last section gives conclusions of the paper and further contemplates the parameters that affect the systems performance.

\section{Simulation model}

The example of simulation scenario is illustrated in fig 1 . The cell with one BS is enhanced by four symmetrically positioned fixed RSs. The simulation process is divided into 40 steps. In every step, one SS joins the network and after that throughput is computed. Thus, model includes 40 SSs which coordinates are pseudo randomly chosen so that $8 \mathrm{SSs}$ are associated to the BS and other 32 are evenly distributed to each RS.

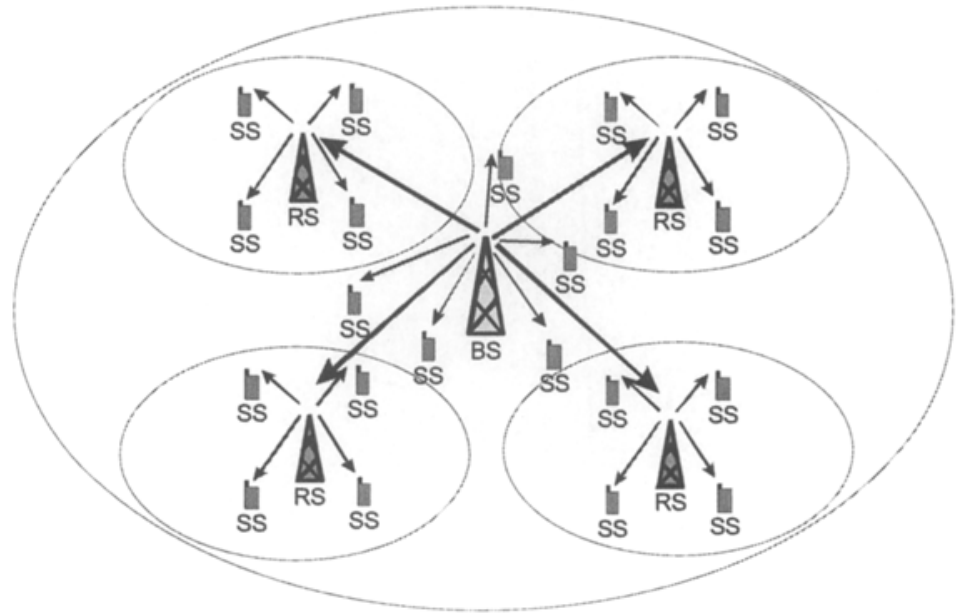

Fig. 1. Simulation scenario

The SSs establish link to the BS or RS according to received SNR. This means that SS try to join to station with the best signal. The BS and RS coverage depends especially on transmitted power, noise, height of antenna above the ground and finally 
on the path loss between the transmitter and the receiver. All necessary parameters used during the simulation process are summarized in Table 1.

Table 1. Simulation parameters

\begin{tabular}{|c|c|c|}
\hline Parameter & \multicolumn{2}{|c|}{ Value } \\
\hline BS range radius (m) & \multicolumn{2}{|c|}{2600} \\
\hline $\mathrm{RS}$ range radius $(\mathrm{m})$ & \multicolumn{2}{|c|}{1000} \\
\hline BS-RS distance $(m)$ & \multicolumn{2}{|c|}{1470} \\
\hline Frequency band $(\mathrm{GHz})$ & \multicolumn{2}{|c|}{5} \\
\hline Number of BS & \multicolumn{2}{|c|}{1} \\
\hline Number of RS & \multicolumn{2}{|c|}{4} \\
\hline Number of SS & \multicolumn{2}{|c|}{40} \\
\hline Channel bandwidth (MHz) & 3.5 & 20 \\
\hline Frame duration (ms) & \multicolumn{2}{|c|}{20} \\
\hline Symbol useful time $t_{b}(\mu s)$ & 64 & 11.64 \\
\hline $\mathrm{t}_{\mathrm{g}} / \mathrm{t}_{\mathrm{b}}$ & $1 / 16$ & $1 / 4$ \\
\hline CP time $t_{g}(\mu s)$ & 4 & 2.91 \\
\hline Symbol time $t_{s}(\mu s)$ & 68 & 15.55 \\
\hline Number of OFDM subcarriers & \multicolumn{2}{|c|}{256} \\
\hline Number of data subcarriers & \multicolumn{2}{|c|}{192} \\
\hline BS transmit power $P_{t}(d B m)$ & \multicolumn{2}{|c|}{30} \\
\hline RS transmit power $P_{t}(\mathrm{dBm})$ & \multicolumn{2}{|c|}{26} \\
\hline BS height $(\mathrm{m})$ & \multicolumn{2}{|c|}{30} \\
\hline RS height $(\mathrm{m})$ & \multicolumn{2}{|c|}{25} \\
\hline SS height $(\mathrm{m})$ & \multicolumn{2}{|c|}{2} \\
\hline $\mathrm{a}$ & \multicolumn{2}{|c|}{3.6} \\
\hline $\mathrm{b}\left(\mathrm{m}^{-1}\right)$ & \multicolumn{2}{|c|}{0.005} \\
\hline $\mathrm{c}(\mathrm{m})$ & \multicolumn{2}{|c|}{20} \\
\hline Noise (dBm) & -108.54 & -100.97 \\
\hline
\end{tabular}

Table 2 demonstrates which type of modulation and coding rate is used in relation to received SNR [1]. Simultaneously, the number of SSs per each burst type is shown for both cases, with no RSs and with RSs. It is evident, that higher modulation orders are seldom utilized for its high SNR requirements. Consequently, the system throughput is largely degraded. But the unfavorable situation is largely improved by RSs utilization. Finally, Table 2 denotes how many bits can be allocated to one OFDM symbol. This information is vital to system capacity estimation. 
Table 2. Type of modulation and coding rate according to SNR

\begin{tabular}{|c|c|c|c|c|c|}
\hline \multirow{2}{*}{\multicolumn{2}{|c|}{$\begin{array}{l}\text { Modulation and } \\
\text { coding rate }\end{array}$}} & \multirow{3}{*}{$\begin{array}{c}\text { Received } \\
\text { SNR (dB) }\end{array}$} & \multicolumn{2}{|c|}{ Number of SSs } & \multirow{3}{*}{$\begin{array}{c}\begin{array}{c}\text { Bits/ } \\
\text { symbol }\end{array} \\
96 \\
\end{array}$} \\
\hline & & & \multirow{2}{*}{$\begin{array}{c}\begin{array}{c}\text { Without } \\
\text { RSs }\end{array} \\
4 \\
\end{array}$} & \multirow{2}{*}{$\begin{array}{c}\text { With } \\
\text { RSs }\end{array}$} & \\
\hline$B P S K$ & $1 / 2$ & & & & \\
\hline \multirow{2}{*}{$Q P S K$} & $1 / 2$ & 6 & 12 & 0 & 192 \\
\hline & $3 / 4$ & 8.5 & 8 & 9 & 288 \\
\hline \multirow{2}{*}{$16 Q A M$} & $1 / 2$ & 11.5 & 12 & 11 & 384 \\
\hline & $3 / 4$ & 15 & 4 & 16 & 576 \\
\hline \multirow{2}{*}{$64 Q A M$} & $2 / 3$ & 19 & 0 & 4 & 768 \\
\hline & $3 / 4$ & 21 & 0 & 0 & 864 \\
\hline
\end{tabular}

During the simulation process, two basic scenarios were considered: i) without RSs and ii) with RSs (case 1). The first one reflects the situation when no RSs are introduced. This scenario may correspond to classical IEEE 802.16-2004 or IEEE 802.16e deployment. The second scenario introduces four RSs where every RS has dedicated own part of MAC frame for its $2^{\text {nd }}$ hop transmission.

However, for a scenario with great number of relays is not profitable to reserve for every RSs transmissions individual part of the frame. The reason is that data transmitted on the $2^{\text {nd }}$ or higher hops increase overhead. Thus, three additionally scenarios were investigated that study its reduction possibility: i) with RSs (case 2) two RSs are allowed to transmit at once, ii) with RSs (case 3) - all active RSs are allowed to transmit at once and iii) with RSs (case 4) - all active RSs may transmit simultaneously and no interference is taken into account. This situation may occur when individual RSs are either in sufficient distance to each other or when some kind of obstacles are between them, e.g. trees, hill, etc.

Before the description of the simulator, it is important to report the assumptions under which the model was developed. These are following: i) all nodes are fixed ii) only DL transmissions are taken into account. But if we assume for the sake of simplicity, that transmit power of SS is the same as BS (RS) or its lower transmit power is compensated by higher BS (RS) received antenna gain, the results may also correspond to UL or combined UL/DL direction, iii) PMP network topology is assumed. Hence, all traffic always leads from the BS via direct path or via one intermediate RS (no communication between SSs is countenance), iv) TDD MAC frame is used.

\section{Simulator description}

All simulation and throughput estimation were generated in Matlab system. Fig. 2 describes the process of simulation step by step. First of all, the input simulation parameters are inserted (see Table 1) and derived parameters are computed. After association of the SS into the network, SNR calculation, number of data OFDM symbols and system throughput is estimated. 


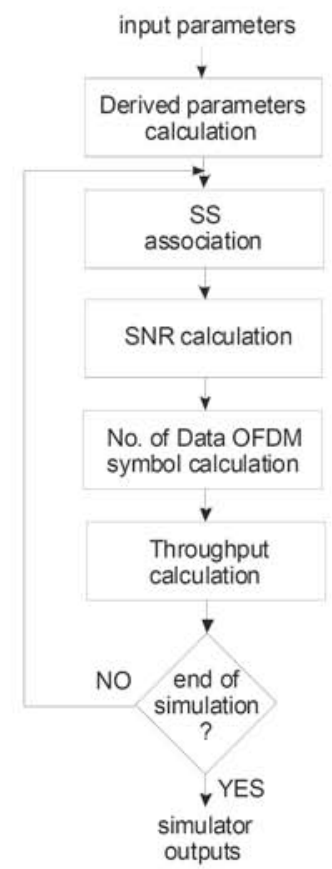

Fig 2. Description of simulation process

\subsection{Path loss model characterization and received SNR calculation}

Path loss estimation Channel model used in simulation was developed by Stanford University and was picked up by its suitability for fixed broadband wireless access [6]. The basic path loss equation with correction factors is calculated as,

$$
P L=A+10 \gamma \log _{10}\left(\frac{d}{d_{o}}\right)+X_{f}+X_{h} .
$$

where, $d$ is the distance between the transmitter and the receiver antennas in meters, $d_{0}=100 \mathrm{~m}$. The other parameters are defined as,

$$
\begin{aligned}
& A=20 \log _{10}\left(\frac{4 \pi d_{0}}{\lambda}\right) . \\
& \gamma=a-b h_{b}+c / h_{b} .
\end{aligned}
$$


where $h_{b}$ is base station height above ground in meters and constant $a, b$ and $c$ are given in Table $\mathrm{xx}$. The correction factors for the operating frequency and for SS height are given as,

$$
\begin{gathered}
X_{f}=6.0 \log _{10}\left(\frac{f}{2000}\right) . \\
X_{h}=-20 \log _{10}\left(\frac{h_{r}}{2}\right) .
\end{gathered}
$$

where $f$ is the frequency in $\mathrm{MHz}$ and hr is the SS antenna height above ground in meters. Further, the received signal strength can by estimated by following equation,

$$
P_{r}=P_{t}-P L \text {. }
$$

where $P_{t}$ is the transmitted signal strength at the side of a receiver. The SNR is computed as,

$$
S N R=P_{r}-\text { Noise } .
$$

where Noise represents either only thermal noise (see Table 1) when no interference is introduced, or summation of thermal noise and interference received by disturbers.

\subsection{Calculation of number of OFDM symbols used for data transmission}

Fig. 3 demonstrates how the throughput of the system is calculated. The overall number of OFDM symbols (variable $N$ ) in one MAC frame is derived from two parameters, i.e. length of OFDM symbol and frame MAC duration. According the Fig. 3, the number of OFDM symbols used only for data is calculated by following formula:

$$
B S \_O F D M=N-\left(X_{1}+X_{2}+X_{3}+X_{4}+X_{5}\right) .
$$

where:

- $\quad N$ is overall number of OFDM symbols in MAC frame.

- $X_{l}$ represents BS broadcast part include long preamble (2 OFDM symbols), frame control header ( 1 OFDM symbol) and finally DL/UL MAPs. Since the broadcast part of the frame is transmitted by the most robust modulation type and coding rate, only 92 bits/OFDM can be carried (see Table 2). The length of the maps and simultaneously the quantity of OFDM symbols depends on the number of IEs in MAPs.

- $X_{2}$ is a gap between DL and UL subframes depends mainly on the round trip delay (for this case 1 OFDM symbol is taken).

- $X_{3}$ are contention slots are composed from initial ranging slot and BW requests slots. The first one takes 5 OFDM symbols to overcome round trip delay and to 
allow transmit ranging request message and long preamble. The $\mathrm{BW}$ request slot is 2 OFDM symbols long (short preamble and BW request message). The quantity of these slots is dependent on the number of users. It is supposed that more users need more slots, thus for every five SSs is dedicated one BW request slot.

- $X_{4}$ is before every UL burst, short preamble (1 OFDM symbol) is appended.

- $X_{5}$ is a gap between the UL and DK subframes (same as $X_{2}$ ).

Calculation of throughput for cases with RSs is slightly more complex (see Fig. 4). Compared with previous scenario, more OFDM symbols are taken for MAC overhead and that is by RS broadcast part of the frame $\left(X_{6}\right)$. The quantity of OFDM symbols used for users data can be computed by following equation:

$$
R S_{-} O F D M=N-\left(X_{1}+X_{2}+X_{3}+X_{4}+X_{5}+X_{6}\right) \text {. }
$$

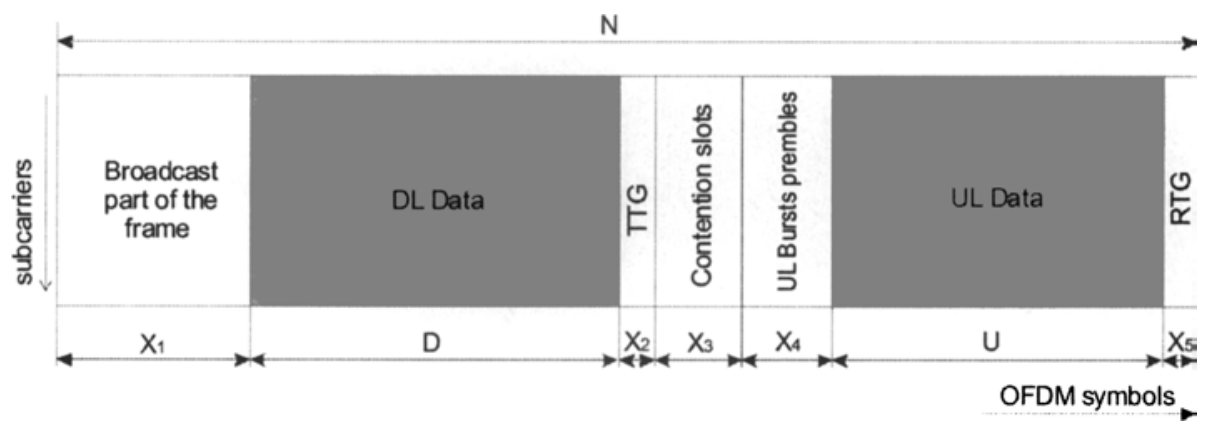

Fig 3. Calculation of OFDM symbol used for data (no RSs)

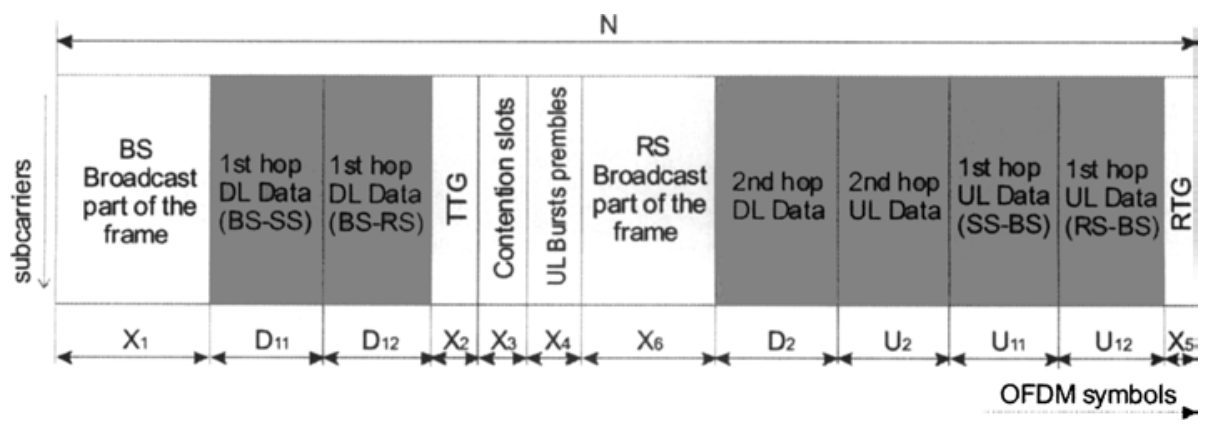

Fig 4. Calculation of OFDM symbol used for data (with RSs) 


\subsection{Throughput calculation}

The rest of the OFDM symbols (BS_OFDM or RS_OFDM) are used for data transmission. The overhead introduced by other eventually MAC messages as well as by MAC PDU headers is neglected since the impact on overall throughput is meaningless. System capacity now depends on the modulation type and coding rate that can be applied to single SSs (according to received SNR). The WiMAX capacity to requested capacity can be estimated by equation 10 for cases without RSs and by equation 11 for cases with RSs:

$$
\begin{aligned}
& W C_{-} t_{-} R C(B S)=\frac{B S_{-} O F D M}{R_{-} O F D M(B S)} . \\
& W C_{-} t_{-} R C(R S)=\frac{R_{-} O F D M}{R_{-} O F D M(R S)} .
\end{aligned}
$$

where $R_{-} O F D M(B S)$ and $R_{-} O F D M(R S)$ represent how many OFDM symbol is needed to transfer given nominal bit rates for all active users. The WiMAX system is able to meet users' requirements as long as the $W_{-} C_{-}{ }_{-} R C$ is higher than one. The $R \_O F D M(B S)$ can be estimated by the following formula,

$$
R_{-} O F D M(B S)=B R^{*} L o F^{*}\left(\sum_{i=1}^{7} \frac{S S G 1_{i}}{b p s_{i}}\right)
$$

where $B R$ is the nominal bit rate in bits, $L o F$ is frame duration in seconds, $S S G 1_{i}$ represent the number of SSs that use individual modulation type and coding rate and finally $b p s_{i}$ express how many bits can be allocated to one OFDM symbol (see Table 2). The calculation of R_OFDM(RS) is computed by:

$$
R_{-} O F D M(R S)=B R^{*} L o F^{*}\left(\sum_{i=1}^{7} \frac{S S G 1_{i}}{b p s_{i}}+\sum_{i=1}^{7} \frac{S S G 2_{i}}{b p s_{i}}+\frac{1}{b p s} \sum_{i=1}^{7} S S G 2_{i}\right) .
$$

where $S S G I_{i}$ has the same meaning like in previous case (number of SSs direct connected to the BS, the $S S G 2_{i}$ corresponds to the quantity of SSs on the $2^{\text {nd }}$ hop (between the RS and SS). The last term in the round bracket represents the transmission between the BS and RS and so bps express the quantity of bits that can be allocated to one OFDM symbol (dependent on the RS-BS link quality).

The throughput per users can be derived from equation 14 for "only BS" scenario and equation 15 for "with RSs" scenarios.

$$
B R=\frac{R \_O F D M(B S)}{L o F^{*}\left(\sum_{i=1}^{7} \frac{S S G 1_{i}}{b p s_{i}}\right)}
$$




$$
B R=\frac{R-\operatorname{OFDM(RS)}}{L o F^{*}\left(\sum_{i=1}^{7} \frac{S S G 1_{i}}{b p s_{i}}+\sum_{i=1}^{7} \frac{S S G 2_{i}}{b p s_{i}}+\frac{1}{b p s} \sum_{i=1}^{7} S S G 2_{i}\right)}
$$

This process is repeated until the end of simulation when outputs of the simulation are obtained by means of results and graphs.

\section{Performance evaluation}

The overall system performance may be measured by its throughput. In Fig. 5 is shown how the throughput per user is decreased when number of SSs grows. Fig. 5a further indicates that for $3.5 \mathrm{MHz}$ channel size the performance with or without RSs is nearly identical.

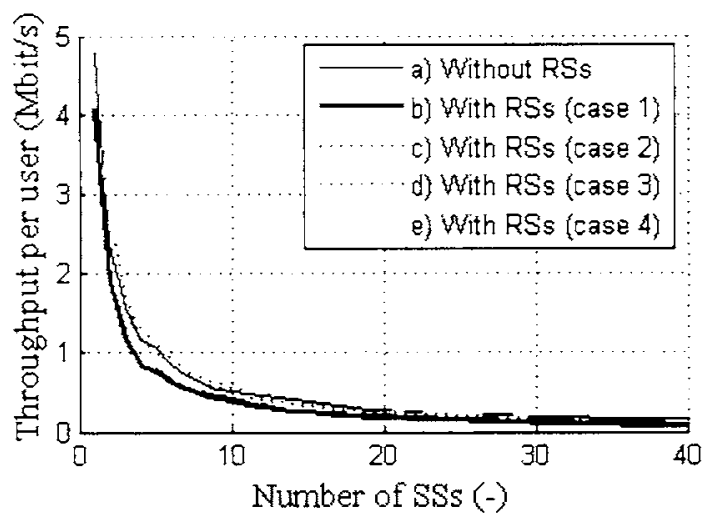

a)

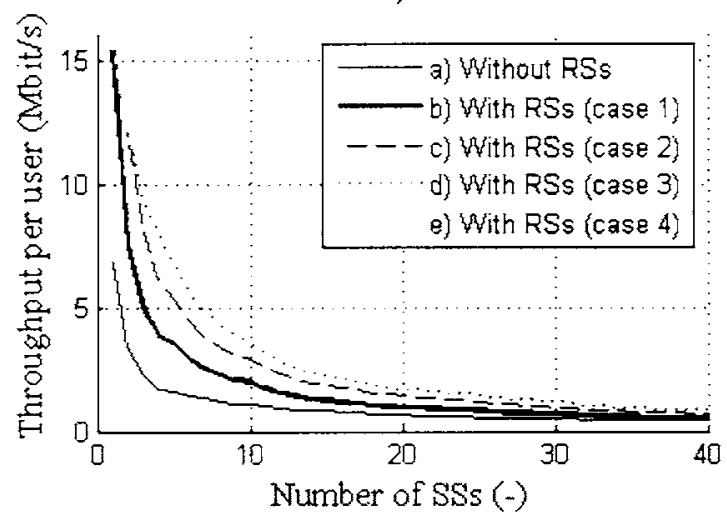

b)

Fig. 5. System throughput per user for: a) $3.5 \mathrm{MHz}$ channel size, b) $20 \mathrm{MHz}$ channel size 
This implies that for fully utilization of relay station merits, the use of wider channel is necessary. If this condition is satisfied, the system capacity may be significantly enhanced, e.g. for 10 active users, the bit rate per user is improved from 1.6 Mbit/s up to $4 \mathrm{Mbit} / \mathrm{s}$ depending on the case used (see Fig. 5b). In general, the improvement strongly rests on the distribution of SSs over the area and its number. Thus the positions of RS must be properly chosen.

Fig. 6 shows system behavior when nominal bit rates are set and the number of SSs is increasing. For reasons listed above, only $20 \mathrm{MHz}$ channel size was taken into consideration. At $y$ axis, the rate of the system to the requested capacity is computed. The capacity limit is denoted by the bold line. If the curve is above the bold line, the free resources are still available and more users can use given nominal bit rate. In opposite, some restrictions have to be applied to support more users. Expectably, the system performance with RSs is better, e.g. nominal bit rate $1 \mathrm{Mbit} / \mathrm{s}$ can be applied up to 35 users against 11 users for system without RSs, before congestion occurs.

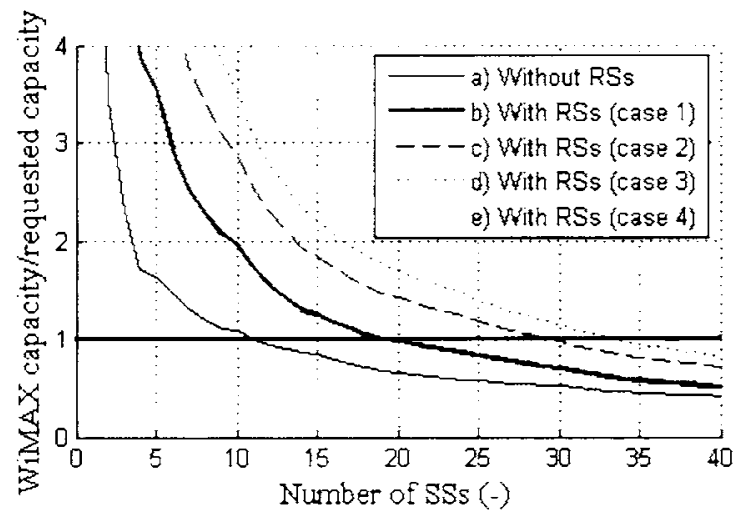

a)

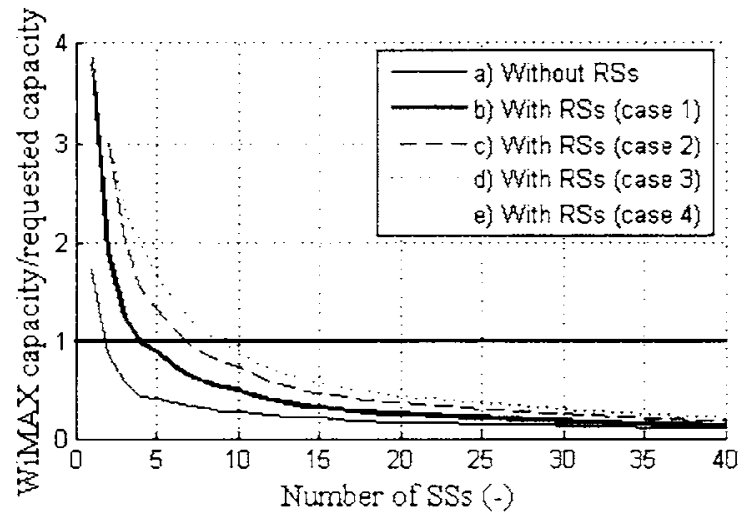

b)

Fig. 6. System throughput for different nominal bit rate: a) $1 \mathrm{Mbit} / \mathrm{s}, \mathrm{b}) 3 \mathrm{Mbit} / \mathrm{s}$ (20 $\mathrm{MHz}$ channel size) 
Other important standpoints that must be taken into account is the height and the transmit power of RSs. The main reason is to avoid of co-channel interference and eventually also disturbance into adjacent channel. How the performance of the system is affected by adjustment of these parameters is shown in Fig. 7 and 8.

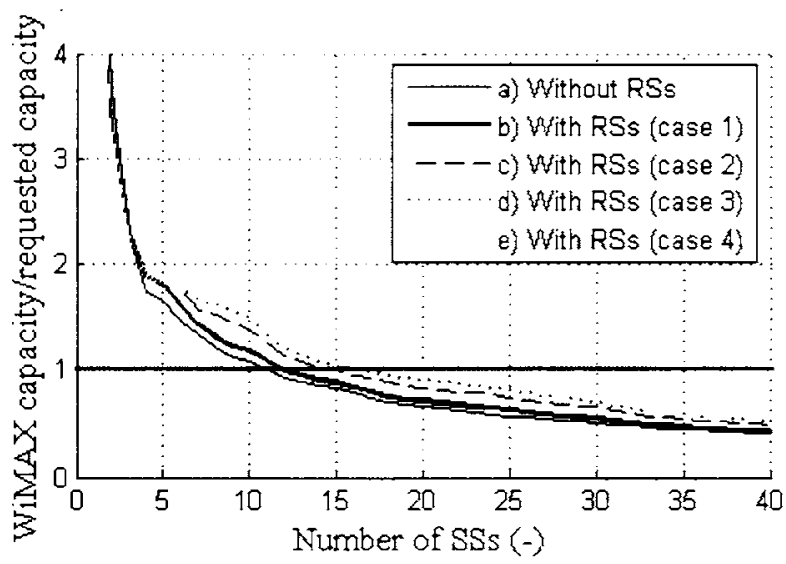

Fig. 7. System performance for RS height of $15 \mathrm{~m}(20 \mathrm{MHz}$ channel size, nominal bit rate $1 \mathrm{Mbit} / \mathrm{s}$ )

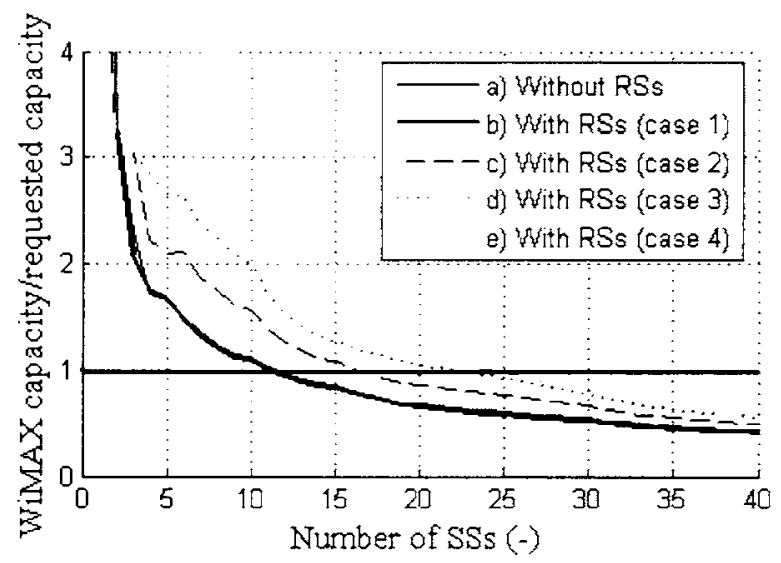

Fig. 8. System performance for RS transmit power $20 \mathrm{dBm}(20 \mathrm{MHz}$ channel size, nominal bit rate $1 \mathrm{Mbit} / \mathrm{s}$ )

It is plain that lowering of RS antenna height as well as reduction of its transmitted power has a great impact on the system performance. This is mainly caused by two facts namely i) RS area coverage is decreased and ii) the link between RS and BS is largely degraded. It could be observed that especially results with RS antenna height $15 \mathrm{~m}$ above the ground are almost comparable to scenario where no RSs were introduced. 


\section{Conclusions}

The paper analyzed the performance of WiMAX system when Relay stations are employed. It was shown that for RSs deployment and their fully merits utilization, several aspects must be carefully considered for their effect on the system performance and that is i) height of RS antenna and its transmit power, ii) numbers of RSs transmit at once and iii) channel size.

The height of RS together with its transmit power have major impact at the system capacity by specifying of RS coverage area and link quality between the BS and RS. The BS-RS transmissions can be considered as overhead. The prospective possibility is to allow more than one RS transmission at the same time. The simulation results support this statement and show that throughput of the system is largely improved when more RSs transmit at once. So the time interval dedicated to the RSs can be significantly reduced. The improvement of the system capacity could be accomplished despite of interference rise.

At last, the results demonstrate that narrow channel size is not suitable for system with RSs. The main reason is that narrow channel size (e.g. $3.5 \mathrm{MHz}$ ) is distinguished by small number of OFDM symbols in MAC frame. Especially, this is true for shorter time MAC frame duration, e.g. $2.5 \mathrm{~ms}$ or $5 \mathrm{~ms}$. In that case, big portion of frame is dedicated for BS-RS transmissions. As result, the overhead introduced by the RSs is much more significant. But this is not the case for the wider channel size (e.g. $20 \mathrm{MHz}$ ) where the percentage of OFDM symbols used for RS is insignificant.

Acknowledgments. This work has been performed in the framework of the FP6 project FIREWORKS IST-27675 STP, which is funded by the European Community. The Authors would like to acknowledge the contributions of their colleagues from FIREWORKS Consortium (http://fireworks.intranet.gr). Further, this research work was supported by Czech Technical University's grant No.CTU0715013.

\section{References}

1. IEEE Std 802.16e-2005, IEEE Standard for Local and metropolitan area networks, Part 16: Air Interface for Fixed and Mobile Broadband Wireless Access Systems, Amendment for Physical and Medium Access Layers for Combined Fixed and Mobile Operation in Licensed Bands, February 2006.

2. A. Molisch, "Wireless Communication", first edition, ISBN 0470848871, November 2005

3. J. Sydir, IEEE 802.16 Broadband Wireless Access Working Group - Harmonized Contribution on 802.16j (Mobile Multihop Relay) Usage Models, July 2006.

4. A. Nosratinia, T. E. Hunter, A. Hedayat, "Cooperative Communication in Wireless Networks", IEEE Communications Magazine, vol. 42, no. 10, October 2004, pp. 68-73.

5. Ch. Hoymann, K. Klagges, "MAC Frame concepts to Support Multihop Communication in IEEE 802.16 Networks", Wireless Word Research Forum, 2005

6. V.S. Abhayawardhana, et al., "Comparison of empirical proagation path loss models for fixed wireless access systems", IEEE Vehicular Technology Conference, 2005. 